\title{
Paracrine control of endothelial cell survival
}

\author{
Dario C. Altieri \\ Boyer Center for Molecular Medicine, Yale University School of Medicine, 295 Congress Avenue, New Haven, \\ Connecticut 06536, USA. Phone: (203) 737-2869; Fax: (203) 737-2402; E-mail: dario.altieri@yale.edu.
}

Apoptosis is generally defined as a genetic program that eliminates unneeded, senescent, or damaged cells. It has attracted tremendous interest from biologists at large for its essential role in development, organ differentiation, and the constant monitoring of homeostasis in the adult organism (1). Cell death/viability pathways exhibit a fascinating complexity. This involves intertwined gene families of stimulators and inhibitors of cell death; biochemical control of mitochondrial homeostasis; and cascade activation of executioner cysteine proteases, caspases (2). It is known that deregulation of apoptosis causes, or at least contributes to, the pathogenesis of human diseases (3). What is less known, despite the several thousands of scientific publications, is how the apoptotic balance is regulated under physiologic or quasi-physiologic conditions to maintain normal homeostasis.

The preservation of endothelial cell viability is one of the clearest examples of how flexible apoptotic mechanisms must be to preserve homeostasis. Our ability to successfully fight infections depends on the recruitment of activated leukocytes at the site of bacterial invasion. This is facilitated by proinflammatory changes occurring in endothelial cells, which respond to cytokines and inflammatory mediators by upregulating leukocyte adhesion-promoting molecules and transcribing their own chemotactic/inflammatory genes (4). The problem is that the same cytokines that elicit these responses (i.e., TNF- $\alpha$ ) are also powerful inducers of apoptosis. How, then, does the endothelium protect itself from committing suicide every time it participates in inflammation? The issue is even more dramatic, because increased endothelial cell apoptosis contributes to vascular diseases with subtle or elusive inflammatory components, such as atherosclerosis or ischemia-reperfusion $(5,6)$. An important mechanism by which endothelial cells can withstand inflammatory challenges is by upregulating several protective, antiapoptotic genes through TNF$\alpha / \mathrm{NF \kappa B}$ activation (7). Therefore, the same transcriptional mechanisms medi- ating inflammation also prevent cytokine-induced cell death, blunting caspase activity and opposing further NFKB activation (7).

However, the recent report by Olofsson et al. (8) adds an unexpected piece to the puzzle of endothelial cell cytoprotection in inflammation. As a non-catalytically active member of chymotrypsinlike leukocyte proteases, heparinbinding protein (HBP) stimulated leukocyte chemotaxis and activation, in addition to its association with heparin. In their study, Olofsson et al. (8) asked whether leukocyte-released HBP might also somehow affect endothelial cell functions. The results have led the authors to a novel model of paracrine control of apoptosis in endothelium. Once released after leukocyte degranulation induced by inflammatory stimuli, HBP bound to endothelial cells through membrane proteoglycans, including syndecans. This was followed by efficient internalization and redistribution of HPB to a subcellular compartment, biochemically and morphologically identifiable as mitochondria. This response diminished caspase- 3 activity and prevented endothelial cell apoptosis induced by growth factor withdrawal (8). Several aspects of this model are intriguing and of potential significance for homeostasis of the vessel wall. First, the structural resemblance to leukocyte elastase and cathepsin G suggests that HBP may have evolved this paracrine cytoprotective function to balance primordial proinflammatory properties. Second, despite the somewhat generic recognition by multiple species of surface proteoglycans, the binding of HPB to endothelial cells must couple to highly specialized mechanism(s) of intracellular trafficking, delivering most, if not all, of approximately $28 \mathrm{kDa}$ intact HBP to a mitochondrial compartment. Third, the reduction in caspase- 3 activity by internalized HPB suggests that this mechanism may afford a broad antiapoptotic spectrum, potentially counteracting other death-inducing stimuli.

Still, several tantalizing questions remain about the pathway (8). First, what does HPB really do in mitochondria? We assume from the notion of mitochondrial catastrophe accompanying apoptosis (9) that it is this subcellular localization that is required for HBP-dependent cytoprotection. However, this point has not yet been demonstrated. And in the mitochondria, does HBP interfere with the upstream initiation of apoptosis, mimicking a bcl-2-like suppression of cytochrome $c$ release and permeability transition $(10,11)$ ? And finally, if this mechanism really preserves endothelial cell viability during inflammation, can HBP block apoptosis induced by TNF$\alpha /$ caspase-8, a pathway that, depending on the cell type, may not even involve perturbation of mitochondrial function? Given the fast-paced field of apoptosis, the response to some of these questions may be forthcoming in the near future. Until then, the study by Olofsson et al. (8) provides a stimulating example of "intercellular collaboration," in which a paracrine cytoprotection by HBP may cooperate with NFKB-inducible protective genes (7) to maintain endothelial cell viability during inflammation.

1. Vaux, D.L., and Korsmeyer, S.J. 1999. Cell death in development. Cell. 96:245-254.

2. Raff, M. 1998. Cell suicide for beginners. Nature. 396:119-122.

3. Thompson, C.B. 1995. Apoptosis in the pathogenesis and treatment of disease. Science. 267:1456-1462.

4. Cines, D.B., et al. 1998. Endothelial cells in physiology and pathophysiology of vascular disorders. Blood. 91:3527-3561.

5. Olivetti, G., et al. 1997. Apoptosis in the failing human heart. N. Engl. J. Med. 336:1131-1141.

6. Bjorkerud, S., and Bjorkarud, B. 1996. Apoptosis is abundant in human atherosclerotic lesions, especially in inflammatory cells (macrophages and $T$ cells), and may contribute to the accumulation of gruel and plaque instability. Am. J. Pathol. 149:367-380.

7. Bach, F.H., Hancock, W.W., and Ferran, C. 1997. Protective genes expressed in endothelial cells: a regulatory response to injury. Immunol. Today. 18:483-486.

8. Olofsson, A.M., et al. 1999. Heparin-binding protein targeted to mitochondrial compartments protects endothelial cells from apoptosis. J. Clin. Invest. 104:885-894.

9. Green, D.R., and Reed, J.C. 1998. Mitochondria and apoptosis. Science. 281:1309-1312.

10. Reed, J.C. 1997. Double identity for proteins of the Bcl-2 family. Nature. 387:773-776.

11. Adams, J.M., and Cory, S. 1998. The Bcl-2 protein family: arbiters of cell survival. Science. 281:1322-1326 